\title{
Maximum Principle for the Space-Time Fractional Conformable Differential System Involving the Fractional Laplace Operator
}

\author{
Tingting Guan (i) and Guotao Wang (i) \\ School of Mathematics and Computer Science, Shanxi Normal University, Linfen 041004, Shanxi, China \\ Correspondence should be addressed to Guotao Wang; wgt2512@163.com
}

Received 25 May 2020; Accepted 29 September 2020; Published 5 November 2020

Academic Editor: Nan-Jing Huang

Copyright (c) 2020 Tingting Guan and Guotao Wang. This is an open access article distributed under the Creative Commons Attribution License, which permits unrestricted use, distribution, and reproduction in any medium, provided the original work is properly cited.

In this paper, the authors consider a IBVP for the time-space fractional PDE with the fractional conformable derivative and the fractional Laplace operator. A fractional conformable extremum principle is presented and proved. Based on the extremum principle, a maximum principle for the fractional conformable Laplace system is established. Furthermore, the maximum principle is applied to the linear space-time fractional Laplace conformable differential system to obtain a new comparison theorem. Besides that, the uniqueness and continuous dependence of the solution of the above system are also proved.

\section{Introduction}

Many fractional partial differential equations were used for modeling complex dynamic systems of engineering, physics, biology, and many other fields [1-4]. As a significant tool, the maximum principle plays an important role in the study of the complex dynamic systems without certain knowledge of the solutions [5-13]. In 2016, by using the maximum principle, Luchko and Yamamoto [14] obtained the uniqueness of both the strong and the weak solutions of the IBVP for a general time-fractional distributed order diffusion equation. In 2016, Jia and $\mathrm{Li}$ [15] applied the maximum principle to the classical solution and weak solution of a time-space fractional diffusion equation. Furthermore, they also deduced the maximum principle for a full fractional diffusion equation other than time-fractional and spatialinteger order diffusion equations. In 2019, Wang et al. [16] investigated the IBVP for Hadamard fractional differential equations with fractional Laplace operator $(-\Delta)^{\beta}$ by using the maximum principle.

There are diverse fractional derivatives, such as the Riemann-Liouville derivative, the Caputo fractional derivative, the left and right conformable derivatives, and other fractional derivatives [17-40]. In 2015, Abdeljawad [34] defined the left and right conformable derivatives.
Depending on [34], Jarad et al. [35] introduced the fractional conformable derivatives and presented the fractional conformable derivative in the sense of Caputo. The extremum principle of the Caputo fractional conformable derivative is seldom regarded in the existing literature. In addition, the papers which mentioned the fractional conformable derivative do not include the fractional Laplace operator.

Motivated by the above works, in this context, the authors investigate the IBVP for a space-time Caputo fractional conformable diffusion system with the fractional Laplace operator. First, we provide a detailed proof of the Caputo fractional conformable extremum principle. Then, the new maximum principle is obtained by applying the extreme principle. As some applications of the maximum principle, a comparison principle for the space-time fractional Laplace conformable differential system is developed, and the properties of the solution of the system are given, such as the uniqueness and continuous dependence on the initial and boundary condition.

The article is organized as follows: in Section 2, the extremum principle for the Caputo fractional conformable derivative is established. In Section 3, the maximum principle of the space-time fractional Laplace conformable differential system is derived, which is used to obtain the comparison principle for the space-time fractional Laplace 
conformable differential system, and the properties of the solution of the above system are given in Section 4 .

\section{Problem Formulation and Extremum Principles}

In this paper, we focus on a space-time Caputo fractional conformable system with the fractional Laplace operator:

$$
\begin{cases}{ }_{a}^{C \beta} D_{t}^{\alpha} u(x, t)+(-\Delta)^{\gamma} u(x, t)-a(x, t) u(x, t)=g(x, t), & (x, t) \in \Omega \times(a, b], \\ u(x, t)=0, & x \in \mathbb{R}^{N} \backslash \bar{\Omega}, t \geq a,\end{cases}
$$

where $\Omega$ represents an open and bounded domain in $\mathbb{R}^{N}(N \geq 1)$ in which boundary $\Gamma$ is smooth and $a(x, t) \in \bar{\Omega} \times[a, b]$ is a bounded function. Here, ${ }_{a}^{C \beta} D_{t}^{\alpha}$ is the left Caputo fractional conformable derivative. For a function $f \in C_{\alpha, a}^{n}$, the left Caputo fractional conformable derivative of order $\beta$ is defined by

$$
{ }_{a}^{C \beta} D_{t}^{\alpha} f(t)=\frac{1}{\Gamma(n-\beta)} \int_{a}^{t}\left(\frac{(t-a)^{\alpha}-(\tau-a)^{\alpha}}{\alpha}\right)^{n-\beta-1} \frac{{ }_{a}^{n} T^{\alpha} f(\tau)}{(\tau-a)^{1-\alpha}} \mathrm{d} \tau,
$$

with $0<\beta<1,0<\alpha<1, n=[\beta]+1,{ }_{a} T^{\alpha} f(t)=(t-a)^{1-\alpha}$ $f^{\prime}(t), \quad{ }_{a}^{n} T^{\alpha}=\underbrace{{ }_{a} T_{a}^{\alpha} T_{a}^{\alpha}, \ldots, T^{\alpha}}_{n \text { times }}$, and $C_{\alpha, a}^{n}[a, b]=\{f$ : $\left.\left.[a, b] \longrightarrow \mathbb{R}\right|_{a} ^{n-1} T^{\alpha} f \in I_{\alpha}[a, b]\right\}$ (where $I_{\alpha}[a, b]$ is defined in Definition 1 of [34]). For detailed information of the Caputo fractional conformable derivative, see [35].

When $\phi \in C_{\text {loc }}^{1,1}\left(\mathbb{R}^{N}\right) \cap L^{\gamma}$, the fractional Laplace operator could be given by

$$
(-\Delta)^{\gamma} \phi(x)=C_{N, \gamma} \int_{\mathbb{R}^{N}} \frac{\phi(x)-\phi(y)}{|x-y|^{N+2 \gamma}} \mathrm{d} y,
$$

with $C_{N, \gamma}=\left(\gamma 2^{2 \gamma} \Gamma(N+2 \gamma / 2) / \pi^{N / 2} \Gamma(1-\gamma)\right)$, and

$$
L_{\gamma}=\left\{\phi: \mathbb{R}^{N} \longrightarrow \mathbb{R} \mid \int_{\mathbb{R}^{N}} \frac{|; \phi(x)|}{1+|x|^{N+\gamma}} \mathrm{d} x<\infty .\right.
$$

Denote

$H(\bar{\Omega})=\left\{u(x, t) \mid u(x, t) \in C^{2,1}(\Omega \times(a, b)), u(x, t) \in C(\bar{\Omega} \times[a, b])\right\}$.

Firstly, we can state two Caputo fractional conformable extremum principles.

Lemma 1. If $f \in C_{\alpha, a}^{1}([a, b])$ reaches its maximum at a point $t_{0} \in[a, b]$, then

$$
{ }_{a}^{C \beta} D_{t_{0}}^{\alpha} f\left(t_{0}\right) \geq 0
$$

holds.

Proof. First, we introduce an auxiliary function

$$
g(t)=f\left(t_{0}\right)-f(t) \geq 0, \quad t \in[a, b] .
$$

Concurrently, $\quad g(t) \in C_{\alpha, a}^{1}([a, b]), \quad g\left(t_{0}\right)=0, \quad$ and ${ }_{a}^{C \beta} D_{t}^{\alpha} g(t)=-{ }_{a}^{C \beta} D_{t}^{\alpha} f(t)$.

By calculation, we notice that

$$
\begin{aligned}
{ }_{a}^{C \beta} D_{\alpha}^{t_{0}} g\left(t_{0}\right)= & \frac{1}{\Gamma(1-\beta)} \int_{a}^{t_{0}}\left(\frac{\left(t_{0}-a\right)^{\alpha}-(\tau-a)^{\alpha}}{\alpha}\right)^{-\beta} \\
& \cdot \frac{(\tau-a)^{1-\alpha} g^{\prime}(\tau)}{(\tau-a)^{1-\alpha} \mathrm{d} \tau} \\
= & \frac{1}{\Gamma(1-\beta)} \int_{a}^{t_{0}}\left(\frac{\left(t_{0}-a\right)^{\alpha}-(\tau-a)^{\alpha}}{\alpha}\right)^{-\beta} g^{\prime}(\tau) \mathrm{d} \tau \\
= & \left.\frac{1}{\Gamma(1-\beta)}\left(\frac{\left(t_{0}-a\right)^{\alpha}-(\tau-a)^{\alpha}}{\alpha}\right)^{-\beta} g(\tau)\right|_{a} ^{t_{0}} \\
& -\frac{\beta}{\Gamma(1-\beta)} \int_{a}^{t_{0}}\left(\frac{\left(t_{0}-a\right)^{\alpha}-(\tau-a)^{\alpha}}{\alpha}\right)^{-\beta-1} \\
& \cdot(\tau-a)^{\alpha-1} g(\tau) \mathrm{d} \tau .
\end{aligned}
$$

This is because

$$
\begin{aligned}
& \lim _{\tau \longrightarrow t_{0}} \frac{1}{\Gamma(1-\beta)}\left(\frac{\left(t_{0}-a\right)^{\alpha}-(\tau-a)^{\alpha}}{\alpha}\right)^{-\beta} g(\tau) \\
& \quad=\frac{\alpha^{\beta}}{\Gamma(1-\beta)} \lim _{\tau \longrightarrow t_{0}} \frac{g^{\prime}(\tau)}{\beta\left(\left(t_{0}-a\right)^{\alpha}-(\tau-a)^{\alpha}\right)^{\beta-1}(-\alpha)(\tau-a)^{\alpha-1}} \\
& \quad=0 .
\end{aligned}
$$

Therefore, formula (9) becomes

$$
\begin{aligned}
{ }_{a}^{C \beta} D_{t_{0}}^{\alpha} g\left(t_{0}\right)= & -\frac{1}{\Gamma(1-\beta)}\left(\frac{\left(t_{0}-a\right)^{\alpha}}{\alpha}\right)^{-\beta} g(a) \\
& -\frac{\beta}{\Gamma(1-\beta)} \int_{a}^{t_{0}}\left(\frac{\left(t_{0}-a\right)^{\alpha}-(\tau-a)^{\alpha}}{\alpha}\right)^{-\beta-1} \\
& \cdot(\tau-a)^{\alpha-1} g(\tau) \mathrm{d} \tau
\end{aligned}
$$

$\leq 0$. 
We can obtain ${ }_{a}^{C \beta} D_{t_{0}}^{\alpha} f\left(t_{0}\right) \leq 0$.

The lemma is proved.

Using the same method, it is easy to obtain the following lemma.

Lemma 2. If $f \in C_{\alpha, a}^{1}([a, b])$ reaches its minimum at a point $t_{0} \in[a, b]$, then

$$
{ }_{a}^{C \beta} D_{t_{0}}^{\alpha} f\left(t_{0}\right) \leq 0
$$

holds.

\section{Maximum Principle}

In this section, we focus on linear space-time Caputo fractional conformable Laplace system (1) with the initialboundary condition:

$$
\begin{aligned}
& u(x, a)=\varphi(x), \quad x \in \Omega, \\
& u(x, t)=\mu(x, t), \quad(x, t) \in \Gamma \times[a, b] .
\end{aligned}
$$

Theorem 1. Let a function $u \in H(\bar{\Omega})$ satisfy linear spacetime Caputo fractional conformable Laplace system (1), (12), and (13). Suppose $g(x, t) \leq 0, \forall(x, t) \in \Omega \times(a, b]$. Then, we have

$$
\begin{array}{r}
u(x, t) \leq \max \left\{\max _{x \in \Omega} \varphi(x), \max _{(x, t) \in \Gamma \times[a, b]} \mu(x, t), 0\right\}, \\
\forall(x, t) \in \bar{\Omega} \times[a, b] .
\end{array}
$$

Proof. We first suppose that inequality (14) is false; then, there exists a point $\left(x_{0}, t_{0}\right) \in \Omega \times(a, b]$ such that

$$
u\left(x_{0}, t_{0}\right)>\max \left\{\max _{x \in \Omega} \varphi(x), \max _{(x, t) \in \Gamma \times[a, b]} \mu(x, t), 0\right\}=M>0 .
$$

Denote $\varepsilon=u\left(x_{0}, t_{0}\right)-M>0$ and

$$
w(x, t)=u(x, t)+\frac{\varepsilon}{2} \frac{b-(t-a)}{b}, \quad(x, t) \in \bar{\Omega} \times[a, b] .
$$

Besides, $w$ implies

$$
\begin{aligned}
w(x, t) & \leq u(x, t)+\frac{\varepsilon}{2}, \quad(x, t) \in \bar{\Omega} \times[a, b] \\
w\left(x_{0}, t_{0}\right) & \geq u\left(x_{0}, t_{0}\right)=\varepsilon+M \geq \varepsilon+u(x, t) \geq \varepsilon+w(x, t)-\frac{\varepsilon}{2} \\
& \geq \frac{\varepsilon}{2}+w(x, t),(x, t) \in(\Gamma \times[a, b]) \cup(\Omega \times\{a\}) .
\end{aligned}
$$

The latter property implies that the maximum of $w$ cannot be attained on $(\Gamma \times[a, b]) \cup(\Omega \times\{a\})$. Let $w\left(x_{1}, t_{1}\right)=\max _{(x, t) \in \bar{\Omega} \times[a, b]} w(x, t)$; then,

$$
w\left(x_{1}, t_{1}\right) \geq u\left(x_{0}, t_{0}\right) \geq \varepsilon+M>\varepsilon
$$

$$
\left.(-\Delta)^{\gamma} w(x, t)\right|_{\left(x_{1}, t_{1}\right)}=C_{N, \gamma} \int_{\mathbb{R}^{N}} \frac{w\left(x_{1}, t_{1}\right)-w\left(x, t_{1}\right)}{\left|x_{1}-x\right|^{N+2 \gamma}} \mathrm{d} x \geq 0 .
$$

By Lemma 1, we know

$$
\left.{ }_{a}^{C \beta} D_{t}^{\alpha} w(x, t)\right|_{\left(x_{1}, t_{1}\right)} \geq 0 .
$$

By calculation, we can show

$$
{ }_{a}^{C \beta} D_{t}^{\alpha}\left(\frac{\varepsilon}{2} \frac{b-(t-a)}{b}\right)=-\frac{1}{\Gamma(1-\beta)} \frac{\varepsilon}{2 b} \int_{a}^{t}\left(\frac{(t-a)^{\alpha}-(\tau-a)^{\alpha}}{\alpha}\right)^{-\beta} \mathrm{d} \tau .
$$

Assuming $u=(\tau-a / t-a)^{\alpha}$ and substituting into formula (21), we get

$$
\begin{aligned}
{ }_{a}^{C \beta} D_{t}^{\alpha}\left(\frac{\varepsilon}{2} \frac{b-(t-a)}{b}\right)= & -\frac{1}{\Gamma(1-\beta)} \frac{\varepsilon}{2 b} \alpha^{\beta-1} \int_{0}^{1}(t-a)^{1-\alpha \beta} \\
& \cdot(1-u)^{-\beta} u^{1-\alpha} \mathrm{d} u \\
= & -\alpha^{\beta-1}(t-a)^{1-\alpha \beta} \frac{\varepsilon}{2 b} \frac{\Gamma(2-\alpha)}{\Gamma(3-\alpha-\beta)} .
\end{aligned}
$$

Applying (19)-(22), it holds that

$$
\begin{aligned}
\left.{ }_{a}^{C \beta} D_{t}^{\alpha} u(x, t)\right|_{\left(x_{1}, t_{1}\right)}+\left.(-\Delta)^{\gamma} u(x, t)\right|_{\left(x_{1}, t_{1}\right)}-a\left(x_{1}, t_{1}\right) u\left(x_{1}, t_{1}\right)-g\left(x_{1}, t_{1}\right) \\
=\left.{ }_{a}^{C \beta} D_{t}^{\alpha} w(x, t)\right|_{\left(x_{1}, t_{1}\right)}-{ }_{a}^{C \beta} D_{t}^{\alpha}\left(\frac{\varepsilon}{2} \frac{b-\left(t_{1}-a\right)}{b}\right)+(-\Delta)^{\gamma} w\left(x_{1}, t_{1}\right) \\
\quad-a\left(x_{1}, t_{1}\right)\left(w\left(x_{1}, t_{1}\right)-\frac{\varepsilon}{2} \frac{b-\left(t_{1}-a\right)}{b}\right)-g\left(x_{1}, t_{1}\right) \\
\geq \alpha^{\beta-1}\left(t_{1}-a\right)^{1-\alpha \beta} \frac{\varepsilon}{2 b} \frac{\Gamma(2-\alpha)}{\Gamma(3-\alpha-\beta)}-a\left(x_{1}, t_{1}\right) \varepsilon\left(1-\frac{b-\left(t_{1}-a\right)}{2 b}\right) \\
\geq 0 .
\end{aligned}
$$


Equation (23) is in contradiction with (1).

The proof of the theorem is completed.

Similarly, the minimum principle can be obtained as follows.

Theorem 2. Let a function $u \in H(\bar{\Omega})$ satisfy linear spacetime Caputo fractional conformable Laplace system (1), (12), and (13). Suppose $g(x, t) \geq 0, \forall(x, t) \in \Omega \times(a, b]$. Then, we have

$$
\begin{array}{r}
u(x, t) \geq \min \left\{\min _{x \in \Omega} \varphi(x), \min _{(x, t) \in \Gamma \times[a, b]} \mu(x, t), 0\right\}, \\
\forall(x, t) \in \bar{\Omega} \times[a, b] .
\end{array}
$$

\section{Some Applications of the Maximum Principle}

Theorem 3. Let $u(x, t) \in H(\bar{\Omega})$ be a solution of system (1) with initial-boundary values (12) and (13). Then,

$$
\begin{aligned}
\|u\|_{C(\bar{\Omega} \times[a, b])} \leq & \max \left\{\max _{x \in \Omega}\|\varphi(x)\|, \max _{(x, t) \in \Gamma \times[a, b]}\|\mu(x, t)\|\right\} \\
& +2 M \frac{\Gamma(2+\alpha \beta-\alpha-\beta)}{\beta \alpha^{\beta} \Gamma(1+\alpha \beta-\alpha)}(b-a)^{\alpha \beta},
\end{aligned}
$$

where

$$
M=\|g\|_{C(\bar{\Omega} \times[a, b])} .
$$

Proof. We first present a function

$$
\begin{array}{r}
w(x, t)=u(x, t)-M \frac{\Gamma(2+\alpha \beta-\alpha-\beta)}{\beta \alpha^{\beta} \Gamma(1+\alpha \beta-\alpha)}(t-a)^{\alpha \beta}, \\
(x, t) \in \bar{\Omega} \times[a, b] .
\end{array}
$$

If $u(x, t)$ is a solution of system (1), (12), and (13), then $w(x, t)$ is a solution of problem (1) with

$$
\begin{aligned}
g_{1}(x, t) & =g(x, t)-M \frac{\Gamma(2+\alpha \beta-\alpha-\beta)}{\beta \alpha^{\beta} \Gamma(1+\alpha \beta-\alpha)}{ }_{a}^{C \beta} D_{t}^{\alpha}(t-a)^{\alpha \beta} \\
& =g(x, t)-M \\
\mu_{1}(x, t) & =\mu(x, t)-M \frac{\Gamma(2+\alpha \beta-\alpha-\beta)}{\beta \alpha^{\beta} \Gamma(1+\alpha \beta-\alpha)}(t-a)^{\alpha \beta} .
\end{aligned}
$$

Substitute $g_{1}(x, t)$ and $\mu_{1}(x, t)$ for $g(x, t)$ and $\mu(x, t)$, respectively. Owing to $g_{1}(x, t) \leq 0$, applying Theorem 1 (maximum principle), we have

$$
\begin{aligned}
w(x, t) \leq & \max \left\{\max _{x \in \Omega}\|\varphi(x)\|, \max _{(x, t) \in \Gamma \times[a, b]}\|\mu(x, t)\|\right. \\
& \left.+M \frac{\Gamma(2+\alpha \beta-\alpha-\beta)}{\beta \alpha^{\beta} \Gamma(1+\alpha \beta-\alpha)}(b-a)^{\alpha \beta}\right\} .
\end{aligned}
$$

Therefore,

$$
\begin{aligned}
u(x, t) \leq & \max \left\{\max _{x \in \Omega}\|\varphi(x)\|, \max _{(x, t) \in \Gamma \times[a, b]}\|\mu(x, t)\|\right\} \\
& +2 M \frac{\Gamma(2+\alpha \beta-\alpha-\beta)}{\beta \alpha^{\beta} \Gamma(1+\alpha \beta-\alpha)}(b-a)^{\alpha \beta} .
\end{aligned}
$$

In a similar manner, we can get

$$
\begin{aligned}
u(x, t) \geq & -\max \left\{\max _{x \in \Omega}\|\varphi(x)\|, \max _{(x, t) \in \Gamma \times[a, b]}\|\mu(x, t)\|\right\} \\
& -2 M \frac{\Gamma(2+\alpha \beta-\alpha-\beta)}{\beta \alpha^{\beta} \Gamma(1+\alpha \beta-\alpha)}(b-a)^{\alpha \beta} .
\end{aligned}
$$

Combining (30) and (31), the theorem is proved.

Theorem 4. Let $u(x, t)$ satisfy $\operatorname{IBVP}(1),(12)$, and (13). $u(x, t)$ is continuous depending on the data given. That is, if

$$
\begin{aligned}
\left\|g-g_{1}\right\|_{C(\bar{\Omega} \times[a, b])} & \leq \varepsilon,\left\|\varphi(x)-\varphi_{1}(x)\right\|_{C(\bar{\Omega})} \\
& \leq \varepsilon_{0},\left\|\mu(x, t)-\mu_{1}(x, t)\right\|_{C(\Gamma \times[a, b])} \leq \varepsilon_{1},
\end{aligned}
$$

then the estimation of the classical solution of $u(x, t)$ and $u_{1}(x, t)$,

$$
\left\|u-u_{1}\right\|_{C(\bar{\Omega} \times[a, b])} \leq \max \left\{\varepsilon_{0}, \varepsilon_{1}\right\}+2 \frac{\Gamma(2+\alpha \beta-\alpha-\beta)}{\beta \alpha^{\beta} \Gamma(1+\alpha \beta-\alpha)}(b-a)^{\alpha \beta} \varepsilon,
$$

holds.

The demonstration process is similar to Theorem 3.

Theorem 5. Let $u \in H(\bar{\Omega})$ be a solution of IBVP (1), (12), and (13). Assume $g(x, t) \leq 0$ and $a(x, t) \leq 0, \forall(x, t) \in \Omega \times$ $(a, b]$. Then, it follows that

$$
u(x, t) \leq 0, \quad(x, t) \in \bar{\Omega} \times[a, b],
$$

if $\varphi(x) \leq 0$, and $\mu(x, t) \leq 0$.

Theorem 6. Let $u \in H(\bar{\Omega})$ satisfy $I B V P(1)$, (12), and (13). Assume $g(x, t) \geq 0$ and $a(x, t) \geq 0, \forall(x, t) \in \Omega \times(a, b]$. Then, it follows that

$$
u(x, t) \geq 0, \quad(x, t) \in \bar{\Omega} \times[a, b],
$$

if $\varphi(x) \geq 0$, and $\mu(x, t) \geq 0$.

The conclusion of Theorem 5 and Theorem 6 is obtained by Theorem 1. 
Remark 1. Let $u \in H(\bar{\Omega})$ satisfy IBVP (1), (12), and (13). Assume $g(x, t)=a(x, t)=0, \forall(x, t) \in \Omega \times(a, b]$. Then, it follows that

$$
u(x, t)=0, \quad \forall(x, t) \in \bar{\Omega} \times[a, b],
$$

if $\varphi(x)=\mu(x, t)=0$.
Theorem 7 (comparison theorem). Suppose $a(x, t) \geq 0$, $b(x, t) \geq 0$, and $b(x, t)>a(x, t), \forall(x, t) \in \Omega \times(a, b]$. Assume $(u, v) \in H(\bar{\Omega}) \times H(\bar{\Omega})$ satisfies the following linear space-time fractional Laplace conformable differential system:

$$
\left\{\begin{array}{l}
{ }_{a}^{C \beta} D_{t}^{\alpha} u(x, t)+(-\Delta)^{\gamma} u(x, t)-a(x, t) v(x, t)-b(x, t) u(x, t) \geq 0, \quad(x, t) \in \Omega \times(a, b], \\
{ }_{a}^{C \beta} D_{t}^{\alpha} v(x, t)+(-\Delta)^{\gamma} v(x, t)-a(x, t) u(x, t)-b(x, t) v(x, t) \geq 0, \quad(x, t) \in \Omega \times(a, b], \\
u(x, t)=0, \quad v(x, t)=0, x \in \mathbb{R}^{N} \backslash \bar{\Omega}, t \geq a, \\
u(x, a) \geq 0, \quad v(x, a) \geq 0, x \in \Omega, \\
u(x, t) \geq 0, \quad v(x, t) \geq 0, \quad(x, t) \in \Gamma \times[a, b] .
\end{array}\right.
$$

Then, it follows that

$$
u(x, t) \geq 0, v(x, t) \geq 0,(x, t) \in \bar{\Omega} \times[a, b] .
$$

Proof. Let $p(x, t)=u(x, t)+v(x, t), \forall(x, t) \in \bar{\Omega} \times[a, b]$. Then, by (37), we have

$$
\left\{\begin{array}{l}
{ }_{a}^{C \beta} D_{t}^{\alpha} p(x, t)+(-\Delta)^{\gamma} p(x, t)-a(x, t) p(x, t)+b(x, t) p(x, t) \geq 0, \quad(x, t) \in \Omega \times(a, b], \\
p(x, t)=0, \quad x \in \mathbb{R}^{N} \backslash \bar{\Omega}, t \geq a, \\
p(x, a) \geq 0, \quad x \in \Omega, \\
p(x, t) \geq 0, \quad(x, t) \in \Gamma \times[a, b] .
\end{array}\right.
$$

Thus, by (39) and Theorem 6, we obtain

Using (37) and (40), we have that

$p(x, t) \geq 0, \quad \forall(x, t) \in \bar{\Omega} \times[a, b]$, i.e.u $(x, t)+v(x, t) \geq 0$,

$$
\cdot(x, t) \in \bar{\Omega} \times[a, b] \text {. }
$$

$$
\begin{aligned}
& \left\{\begin{array}{l}
{ }_{a}^{C \beta} D_{t}^{\alpha} u(x, t)+(-\Delta)^{\gamma} u(x, t)-(b(x, t)-a(x, t)) u(x, t) \geq 0, \quad(x, t) \in \Omega \times(a, b], \\
u(x, t)=0, \quad x \in \mathbb{R}^{N} \backslash \bar{\Omega}, t \geq a, \\
u(x, a) \geq 0, \quad x \in \Omega, \\
u(x, t) \geq 0, \quad(x, t) \in \Gamma \times[a, b],
\end{array}\right. \\
& \begin{cases}{ }_{a}^{C} D_{t}^{\alpha} v(x, t)+(-\Delta)^{\gamma} v(x, t)-(b(x, t)-a(x, t)) v(x, t) \geq 0, \quad(x, t) \in \Omega \times(a, b], \\
v(x, t)=0, & x \in \mathbb{R}^{N} \backslash \bar{\Omega}, t \geq a, \\
v(x, a) \geq 0, & x \in \Omega, \\
v(x, t) \geq 0, & (x, t) \in \Gamma \times[a, b] .\end{cases}
\end{aligned}
$$

Applying Theorem 6 to (41) and (42), we can get

$$
u(x, t) \geq 0, v(x, t) \geq 0,(x, t) \in \bar{\Omega} \times[a, b] .
$$

Thus, the conclusion holds.
Similarly, the following theorem holds.

Theorem 8. Suppose $a(x, t) \leq 0, b(x, t) \leq 0$, and $b(x, t)<$ $a(x, t), \forall(x, t) \in \Omega \times(a, b]$. Assume $(u, v) \in H(\bar{\Omega}) \times H(\bar{\Omega})$ 
satisfies the following linear space-time fractional Laplace conformable differential system:

$$
\begin{cases}{ }_{a}^{C \beta} D_{t}^{\alpha} u(x, t)+(-\Delta)^{\gamma} u(x, t)-a(x, t) v(x, t)-b(x, t) u(x, t) \leq 0, & (x, t) \in \Omega \times(a, b], \\ { }_{a} \beta D_{t}^{\alpha} v(x, t)+(-\Delta)^{\gamma} v(x, t)-a(x, t) u(x, t)-b(x, t) v(x, t) \leq 0, & (x, t) \in \Omega \times(a, b] \\ u(x, t)=0, v(x, t)=0, \quad x \in \mathbb{R}^{N} \backslash \bar{\Omega}, t \geq a, & \\ u(x, a) \leq 0, v(x, a) \leq 0, \quad x \in \Omega, \\ u(x, t) \leq 0, v(x, t) \leq 0, \quad(x, t) \in \Gamma \times[a, b] .\end{cases}
$$

Then, it follows that

$$
u(x, t) \leq 0, v(x, t) \leq 0,(x, t) \in \bar{\Omega} \times[a, b] .
$$

Remark 2. Let $(u, v) \in H(\bar{\Omega}) \times H(\bar{\Omega})$ satisfy the following linear space-time fractional Laplace conformable differential system:

$$
\begin{cases}{ }_{a}^{C} D_{t}^{\alpha} u(x, t)+(-\Delta)^{\gamma} u(x, t)-a(x, t) v(x, t)-b(x, t) u(x, t)=0, & (x, t) \in \Omega \times(a, b], \\ { }_{a} \beta & D_{t}^{\alpha} v(x, t)+(-\Delta)^{\gamma} v(x, t)-a(x, t) u(x, t)-b(x, t) v(x, t)=0, \quad(x, t) \in \Omega \times(a, b], \\ u(x, t)=0, v(x, t)=0, \quad x \in \mathbb{R}^{N} \backslash \bar{\Omega}, t \geq a, & \\ u(x, a)=\varphi_{1}(x), v(x, a)=\varphi_{2}(x), \quad x \in \Omega, \\ u(x, t)=\mu_{1}(x, t), v(x, t)=\mu_{2}(x, t), \quad(x, t) \in \Gamma \times[a, b] .\end{cases}
$$

Suppose $a(x, t)=b(x, t)=0, \forall(x, t) \in \Omega \times(a, b]$. Then, it follows that

$$
u(x, t)=0, v(x, t)=0, \quad \forall(x, t) \in \bar{\Omega} \times[a, b],
$$

if $\varphi_{1}(x)=\varphi_{2}(x)=\mu_{1}(x, t)=\mu_{2}(x, t)=0$.

Next, we focus on the following linear space-time fractional Laplace conformable differential system:

$$
\left\{\begin{array}{l}
{ }_{a}^{C \beta} D_{t}^{\alpha} u(x, t)+(-\Delta)^{\gamma} u(x, t)-a(x, t) v(x, t)-b(x, t) u(x, t)=g_{1}(x, t), \quad(x, t) \in \Omega \times(a, b], \\
{ }_{a}^{C \beta} D_{t}^{\alpha} v(x, t)+(-\Delta)^{\gamma} v(x, t)-a(x, t) u(x, t)-b(x, t) v(x, t)=g_{2}(x, t), \quad(x, t) \in \Omega \times(a, b], \\
u(x, t)=0, v(x, t)=0, \quad x \in \mathbb{R}^{N} \backslash \bar{\Omega}, t \geq a, \\
u(x, a)=\varphi_{1}(x), v(x, a)=\varphi_{2}(x), \quad x \in \Omega, \\
u(x, t)=\mu_{1}(x, t), v(x, t)=\mu_{2}(x, t), \quad(x, t) \in \Gamma \times[a, b] .
\end{array}\right.
$$

Theorem 9. Suppose $a(x, t) \leq 0, b(x, t) \leq 0, b(x, t)<a(x, t)$, $g_{1}(x, t) \leq 0$, and $g_{2}(x, t) \leq 0, \quad \forall(x, t) \in \Omega \times(a, b]$; then,

$$
\begin{array}{r}
u(x, t)=u_{1}(x, t)-u_{2}(x, t), v(x, t)=v_{1}(x, t)-v_{2}(x, t), \\
\forall(x, t) \in \bar{\Omega} \times[a, b],
\end{array}
$$

IBVP (48) has a unique solution on $H(\bar{\Omega}) \times H(\bar{\Omega})$.

Proof. Let $\left(u_{1}, v_{1}\right)$ and $\left(u_{2}, v_{2}\right)$ be two solutions of IBVP satisfies the system (48). Denote

$$
\begin{cases}{ }_{a}^{C \beta} D_{t}^{\alpha} u(x, t)+(-\Delta)^{\gamma} u(x, t)-a(x, t) v(x, t)-b(x, t) u(x, t)=0, & (x, t) \in \Omega \times(a, b], \\ { }_{a}^{C \beta} D_{t}^{\alpha} v(x, t)+(-\Delta)^{\gamma} v(x, t)-a(x, t) u(x, t)-b(x, t) v(x, t)=0, & (x, t) \in \Omega \times(a, b], \\ u(x, t)=0, v(x, t)=0, \quad x \in \mathbb{R}^{N} \backslash \bar{\Omega}, t \geq a, & \\ u(x, a)=0, v(x, a)=0, \quad x \in \Omega, \\ u(x, t)=0, v(x, t)=0, \quad(x, t) \in \Gamma \times[a, b] .\end{cases}
$$


Let $p(x, t)=u(x, t)+v(x, t), \forall(x, t) \in \bar{\Omega} \times[a, b]$. Ву

(50), we have

$$
\left\{\begin{array}{l}
{ }_{a}^{C \beta} D_{t}^{\alpha} p(x, t)+(-\Delta)^{\gamma} p(x, t)-(b(x, t)-a(x, t)) p(x, t)=0, \quad(x, t) \in \Omega \times(a, b] . \\
p(x, t)=0, \quad x \in \mathbb{R}^{N} \backslash \bar{\Omega}, t \geq a, \\
p(x, a)=0, \quad x \in \Omega, \\
p(x, t)=0, \quad(x, t) \in \Gamma \times[a, b] .
\end{array}\right.
$$

Applying Theorem 8, we get

$$
u(x, t) \leq 0, v(x, t) \leq 0,(x, t) \in \bar{\Omega} \times[a, b] .
$$

By the same way, using Theorem 8 to $-u(x, t)$ and $-v(x, t)$, we have

$$
u(x, t) \geq 0, v(x, t) \geq 0,(x, t) \in \bar{\Omega} \times[a, b] .
$$

Combining (52) and (53), we can get

$$
u(x, t)=0, v(x, t)=0, \quad \forall(x, t) \in \bar{\Omega} \times[a, b] .
$$

Thus, the conclusion holds.

\section{Data Availability}

No data were used to support this study.

\section{Conflicts of Interest}

The authors declare no conflicts of interest.

\section{Authors' Contributions}

Both authors contributed equally and approved the final manuscript.

\section{Acknowledgments}

The research work of Guotao Wang was supported by the NSF of Shanxi, China, Project no. 201701D221007.

\section{References}

[1] R. Hilfer, Applicaions of Fractional Calculus in Physica, Word Scietific, Singapore, Singapore, 2000.

[2] A. Kilbas, H. Srivastava, and J. Trujillo, Therory and Appliction of Fractional Differential Equations, North-Holland Mathematics Studies, vol. 247, , 2006.

[3] Z. Wang, Y. Xie, J. Lu, and Y. Li, "Stability and bifurcation of a delayed generalized fractional-order prey-predator model with interspecific competition," Applied Mathematics and Computation, vol. 347, pp. 360-369, 2019.

[4] Z. Wang, X. Wang, Y. Li, and X. Huang, "Stability and Hopf bifurcation of fractional-order complex-valued single neuron model with time delay," International Journal of Bifurcation and Chaos, vol. 27, no. 13, Article ID 1750209, 2017.

[5] L. Zhang, B. Ahmad, and G. Wang, "Analysis and application for a diffusion equations with a new fractional derivative without singular kernel," Electron Journal of Differential Equations, vol. 289, pp. 1-6, 2017.

[6] Y. Luchko and M. Yamamoto, "On the maximum principle for a time-fractional diffusion equations," Fractional Calculus and Applied Analysis, vol. 20, no. 5, pp. 1131-1145, 2017.

[7] Y. Luchko, "Initial-boundary-value problems for the onedimensional time-fractional diffusion equation," Fractional Calculus and Applied Analysis, vol. 15, no. 1, pp. 141-160, 2012.

[8] M. Kirane and B. T. Torebek, "Extremum principle for the Hadamard derivatives and its application to nonlinear fractional partial differential equations," Fractional Calculus and Applied Analysis, vol. 22, no. 2, pp. 358-378, 2019.

[9] Y. Luchko, "Maximum principle and its application for the time-fractional diffusion equations," Fractional Calculus and Applied Analysis, vol. 14, no. 1, pp. 110-124, 2011.

[10] Y. Luchko, "Initial-boundary-value problems for the generalized multi-term time-fractional diffusion equation," Journal of Mathematical Analysis and Applications, vol. 374, no. 2, pp. 538-548, 2011.

[11] M. Al-Refai and Y. Luchko, "Maximum principle for the multi-term time-fractional diffusion equations with the Riemann-Liouville fractional derivatives," Applied Mathematics and Computation, vol. 257, pp. 40-51, 2015.

[12] Y. Luchko, "Boundary value problem for the generalized time-fractional diffusion equation of distributed order," Fractional Calculus and Applied Analysis, vol. 12, pp. 409422, 2009.

[13] Y. Luchko, "Maximum principle for the generalized timefractional diffusion equation," Journal of Mathematical Analysis and Applications, vol. 351, no. 1, pp. 218-223, 2009.

[14] Y. Luchko and M. Yamamoto, "General time-fractional diffusion equation: some uniqueness and existence results for the initial-boundary-value problems," Fractional Calculus and Applied Analysis, vol. 19, no. 3, pp. 676-695, 2016.

[15] J. Jia and K. Li, "Maximum principles for a time-space fractional diffusion equation," Applied Mathematics Letters, vol. 62 , pp. 23-28, 2016.

[16] G. Wang, X. Ren, and D. Baleanu, "Maximum principle for Hadamard fractional differential equations involving fractional Laplace," Mathematical Methods in the Applied Sciences, vol. 43, no. 1, pp. 1-10, 2019.

[17] G. Wang, X. Ren, Z. Bai, and W. Hou, "Radial symmetry of standing waves for nonlinear fractional Hardy-Schrödinger equation," Applied Mathematics Letters, vol. 96, pp. 131-137, 2019.

[18] K. Pei, G. Wang, and Y. Sun, "Successive iterations and positive extremal solutions for a Hadamard type fractional integro-differential equations on infinite domain," Applied Mathematics and Computation, vol. 312, pp. 158-168, 2017. 
[19] G. Wang, "Twin iterative positive solutions of fractional q-difference Schrödinger equations," Applied Mathematics Letters, vol. 76, pp. 103-109, 2018.

[20] G. Wang, "Explicit iteration and unbounded solutions for fractional integral boundary value problem on an infinite interval,” Applied Mathematics Letters, vol. 47, pp. 1-7, 2015.

[21] L. Zhang and W. Hou, "Standing waves of nonlinear fractional p-Laplacian Schrödinger equation involving logarithmic nonlinearity," Applied Mathematics Letters, vol. 102, Article ID 106149, 2020.

[22] G. Wang, K. Pei, and Y. Chen, "Stability analysis of nonlinear Hadamard fractional differential system," Journal of the Franklin Institute, vol. 356, no. 12, pp. 6538-6546, 2019.

[23] L. Zhang, B. Ahmad, G. Wang, and X. Ren, "Radial symmetry of solution for fractional p-Laplacian system," Nonlinear Analysis, vol. 196, Article ID 111801, 2020.

[24] G. Wang, K. Pei, and D. Baleanu, "Explicit iteration to Hadamard fractional integro-differential equations on infinite domain," Advances in Difference Equations, vol. 299, pp. 1-11, 2016.

[25] L. Zhang, N. Qin, and B. Ahmad, "Explicit iterative solution of a Caputo-Hadamard type fractional turbulent flow model," Mathematical Methods in the Applied Sciences, pp. 1-11, 2020.

[26] W. Chen and J. Zhu, "Indefinite fractional elliptic problem and Liouville theorems," Journal of Differential Equations, vol. 260, no. 5, pp. 4758-4785, 2016.

[27] G. Wang, K. Pei, R. P. Agarwal, L. Zhang, and B. Ahmad, "Nonlocal Hadamard fractional boundary value problem with Hadamard integral and discrete boundary conditions on a half-line," Journal of Computational and Applied Mathematics, vol. 343, pp. 230-239, 2018.

[28] L. A. Caffarelli, S. Salsa, and L. Silvestre, "Regularity estimates for the solution and the free boundary of the obstacle problem for the fractional Laplacian," Inventiones Mathematicae, vol. 171, no. 2, pp. 425-461, 2008.

[29] L. Silvestre, "Regularity of the obstacle problem for a fractional power of the Laplace operator," Communications on Pure and Applied Mathematics, vol. 60, no. 1, pp. 67-112, 2007.

[30] B. Barrios, E. Colorado, A. de Pablo, and U. Sánchez, "On some critical problems for the fractional Laplacian operator," Journal of Differential Equations, vol. 252, no. 11, pp. 61336162, 2012.

[31] C. Brändle, E. Colorado, A. de Pablo, and U. Sánchez, "A concave-convex elliptic problem involving the fractional Laplacian," Proceedings of the Royal Society of Edinburgh: Section A Mathematics, vol. 143, no. 1, pp. 39-71, 2013.

[32] W. Chen, Y. Li, and R. Zhang, "A direct method of moving spheres on fractional order equations," Journal of Functional Analysis, vol. 272, no. 10, pp. 4131-4157, 2017.

[33] W. Chen, C. Li, and Y. Li, "A direct method of moving planes for the fractional Laplacian," Advances in Mathematics, vol. 308, pp. 404-437, 2017.

[34] T. Abdeljawad, "On conformable fractional calculus," Journal of Computational and Applied Mathematics, vol. 279, pp. 57-66, 2015.

[35] F. Jarad, E. Uğurlu, T. Abdeljawad, and D. Baleanu, "On a new class of fractional operators," Advances in Difference Equations, vol. 247, pp. 1-16, 2017.

[36] Y.-K. Chang, Y. Pei, and R. Ponce, "Existence and optimal controls for fractional stochastic evolution equations of Sobolev type via fractional resolvent operators," Journal of Optimization Theory and Applications, vol. 182, no. 2, pp. 558-572, 2019.
[37] X.-J. Yang, F. Gao, and H. M. Srivastava, "Exact travelling wave solutions for the local fractional two-dimensional Burgers-type equations," Computers \& Mathematics with Applications, vol. 73, no. 2, pp. 203-210, 2017.

[38] G. Wang and X. Ren, "Radial symmetry of standing waves for nonlinear fractional Laplacian Hardy-Schrödinger systems," Applied Mathematics Letters, vol. 110, Article ID 106560, 2020.

[39] Y. Wang and C. Hou, "Existence of multiple positive solutions for one-dimensional p-Laplacian," Journal of Mathematical Analysis and Applications, vol. 315, no. 1, pp. 144-153, 2006.

[40] J. Qin, G. Wang, L. Zhang, and B. Ahmad, "Monotone iterative method for a $p$-Laplacian boundary value with fractional conformable derivatives," Boundary Value Problems, vol. 145, pp. 1-12, 2019. 